\title{
BMJ Global Health A quality improvement approach to the reduction of HIV-related stigma and discrimination in healthcare settings
}

\author{
Daniel J Ikeda, ${ }^{1}$ Laura Nyblade,${ }^{2}$ Kriengkrai Srithanaviboonchai, ${ }^{3}$ \\ Bruce D Agins ${ }^{\oplus 1,4}$
}

\begin{abstract}
To cite: Ikeda DJ, Nyblade L, Srithanaviboonchai K, et al. A quality improvement approach to the reduction of HIV-related stigma and discrimination in healthcare settings. BMJ Global Health 2019:4:e001587. doi:10.1136/ bmjgh-2019-001587
\end{abstract}

Handling editor Seye Abimbola

Received 28 March 2019 Revised 16 May 2019 Accepted 25 May 2019

Check for updates

(c) Author(s) (or their employer(s)) 2019. Re-use permitted under CC BY-NC. No commercial re-use. See rights and permissions. Published by BMJ.

${ }^{1}$ HEALTHQUAL, Institute for Global Health Sciences, University of California San Francisco, San Francisco, California, USA

${ }^{2}$ Research Triangle Institute, Washington, District of Columbia, USA

${ }^{3}$ Dept of Community Medicine, Faculty of Medicine, Research Institute for Health Sciences, Chiang Mai University, Chiang Mai, Thailand

${ }^{4}$ Department of Epidemiology and Blostatistics, University of California San Francisco, San Francisco, California, USA

Correspondence to Dr Bruce D Agins; bruce.agins@ucsf.edu

\section{ABSTRACT}

HIV-related stigma and discrimination (S\&D) in healthcare settings represents a potent barrier to achieving global aims to end the HIV epidemic, particularly in Southeast Asia (Cambodia, Lao People's Democratic Republic, Thailand and Vietnam). Evidence-based approaches for measuring and reducing S\&D in healthcare settings exist, but their incorporation into routine practice remains limited, in part due to a lack of attention to how unique organisational practices - beyond the knowledge and attitudes of individuals-may abet and reinforce S\&D. Application of a quality improvement (QI) approach in which facilities leverage routine measurement of S\&D among healthcare workers and people living with HIV, team-based learning, root cause analysis, and tests of change offers a novel means through which to address S\&D in local contexts and develop interventions to address individual-level and organisation-level drivers of S\&D. To support the adoption of a QI approach to S\&D reduction, the Southeast Asia Stigma Reduction QI Learning Network was launched with Ministries of Health from Cambodia, Lao PDR, Thailand and Vietnam, to co-develop strategies for implementing Ql activities in participating facilities. Since the inception of Network activities in 2017, Ministry-led Ql activities to address S\&D have been implemented in 83 facilities and 29 provinces across participating countries. Moreover, 27 strategies and interventions have been tested to date and are being evaluated for scale up by participating facilities, spanning multiple drivers and organisational domains. Lessons learned through Network activities offer nationallevel and facility-level HIV programmes best practices for implementing a $\mathrm{Ql}$ approach to $\mathrm{S} \& \mathrm{D}$ reduction.

\section{INTRODUCTION}

The Joint United Nations Programme on HIV/AIDS has developed an ambitious strategy to end AIDS as a global health threat by 2030, with specific aims to ensure that $95 \%$ of people living with HIV (PLWH) know their status, $95 \%$ of those who know their status receive antiretroviral therapy and $95 \%$ of those receiving antiretroviral therapy achieve viral suppression ('95-95-95 targets'). ${ }^{1}$ In Southeast Asia (Cambodia, Lao People's Democratic Republic, Thailand and

\section{Summary box}

- HIV-related stigma and discrimination (S\&D) in healthcare settings undermines efforts to control the HIV epidemic in Southeast Asia (Cambodia, Lao People's Democratic Republic, Thailand and Vietnam) by compromising access to HIV prevention and treatment services.

- Despite the existence of approaches to measure and reduce S\&D, these approaches are not routinely integrated into ongoing efforts to assess, assure and improve the quality of HIV service delivery.

- Applying a quality improvement (Ql) approach to the reduction of HIV-related S\&D offers a novel way to ensure that national-level and facility-level programme routinely measure S\&D and implement interventions to reduce S\&D that are adapted to local contexts.

- Lessons learnt from implementation of the Southeast Asia Stigma Reduction QI Learning Network provide national-level and facility-level programme with strategies to implement a QI approach to stigma reduction, with a focus on how routine measurement, root cause analysis, peer learning and other QI methods can be leveraged to address individual and organisational drivers of $S \& D$.

Vietnam), where an estimated 769000 people were living with HIV in 2017 and antiretroviral therapy in the public sector is rapidly decentralising, ${ }^{1}$ HIV-related stigma and discrimination (S\&D) and intersecting stigma among key populations disproportionately burdened by HIV (eg, men who have sex with men, transgender people, sex workers, people who inject drugs) thwart efforts to achieve these aims.

HIV-related S\&D manifests itself in myriad, intersecting ways, and has been shown to be damaging in the healthcare setting, where PLWH must access essential medical care and interact with numerous cadres of healthcare workers (HCWs), including nurses, doctors and administrative support staff, among others. ${ }^{2}$ In these settings, HIV-related S\&D 
is abetted and reinforced by four immediately actionable drivers: HCW attitudes towards PLWH, HCW fears of contracting HIV in the workplace, HCW awareness of S\&D and the institutional environment. ${ }^{3}$ These drivers promote the development and support of clinical environments that deter willingness to seek testing, prevention and treatment services, ${ }^{4}$ undermine treatment adherence,${ }^{5}$ and promote care disengagement, ${ }^{6}$ thereby compromising full access to the benefits of HIV services. In recognition of the barriers posed by S\&D in the healthcare setting, the 2016 United Nations Political Declaration on Ending AIDS called on member nations to commit to eliminating S\&D in healthcare settings, ${ }^{7}$ and the Association of Southeast Asian Nations has promoted 'Zero Discrimination' as a pillar of the regional drive to end the HIV epidemic. ${ }^{8}$

Despite broad global commitment to end HIV-related S\&D in healthcare settings-and the existence of validated tools ${ }^{9}$ to measure it and evidence-based interventions ${ }^{10-12}$ to address it-efforts to incorporate S\&D-reduction activities into healthcare settings at scale remain limited.$^{13}$ Previous initiatives to reduce S\&D were often limited to training alone and episodic rather than continuous measurement of its prevalence-an approach which is likely insufficient to eliminate S\&D in healthcare settings. To be sure, training has been shown to be effective in bridging gaps in knowledge that may perpetuate $\mathrm{S} \& \mathrm{D},{ }^{11}$ but it does not address other factors through which S\&D is produced and abetted. To be effective, S\&D interventions that target individual attitudes and behaviours must also consider the prevailing processes within healthcare organisations, and how these processes mediate the translation of knowledge gains into outcomes such as reductions in $\mathrm{S} \& \mathrm{D}$ and changes in organisational practice. Unfortunately, few studies to date have explored the effectiveness of organisation-level interventions in reducing $\mathrm{S} \& \mathrm{D},{ }^{13}{ }^{14}$ and little work has been done to translate aspirations of health equity into institutional practice.

As the drivers of HIV-related S\&D in the healthcare setting are multi-dimensional, emergent, highly contextual and engrained in organisational behaviour, ${ }^{15}$ so, too, must S\&D-reduction interventions be multi-faceted, vigilant of change, sensitive to local context and targeted to individuals as well as organisations. Application of quality improvement (QI) methods offers an innovative approach to identify individual-level and system-level root causes of S\&D and mount data-driven, team-based, contextually appropriate responses to address them in a local setting. QI approaches have long been applied to address gaps in HIV clinical outcomes such as care engagement and viral suppression, ${ }^{16}$ yet little work to date has sought to apply these approaches to S\&D reduction. ${ }^{14}$ As national HIV programme monitors the quality of HIV care as part of routine activities, the incorporation of S\&D measurement and intervention into these activities may represent a sustainable means to rapidly scale S\&D-reduction programming.
In 2017, HEALTHQUAL at the University of California, San Francisco, launched the Southeast Asia Stigma Reduction QI Learning Network ('Network') with financial support through the Health Resources and Services Administration as part of the US President's Emergency Plan for AIDS Relief. The aim of the Network was to 'enhance the adoption, implementation, and sustainability ${ }^{17}$ of a QI approach to S\&D reduction into national-level and facility-level HIV programming in Cambodia, Lao People's Democratic Republic, Thailand and Vietnam. These countries face concentrated HIV epidemics characterised by high prevalence among key populations which may face the added stigma of being associated with an already marginalised group (eg, men who have sex with men, transgender people, sex workers, injection drug users) in addition to the stigma associated with HIV. Previous work has documented high levels of HIV-related S\&D in healthcare settings in the region, ${ }^{18-22}$ and key stakeholders from all four countries continue to identify S\&D in the healthcare setting as a barrier to achieving epidemic control. In this work, we describe the application of a QI approach to the reduction of HIV-related S\&D in the healthcare setting, and synthesise lessons learnt through implementation of Network activities. Insights from this work hold implications for HIV programmes as they operationalise global aims to eliminate HIV-related S\&D in healthcare settings, and seek to embed these aims within broader efforts to measure, assure and improve the quality of HIV service delivery.

\section{APPROACH \\ QI approach}

While definitions of QI vary, at the core of any QI approach is the use of routine measurement, group learning, root cause analysis, and insights from organisational psychology, systems thinking, and iterative tests of change to design, implement, and evaluate interventions that improve outcomes. ${ }^{23} \mathrm{QI}$ approaches in healthcare settings have been used to effectively address gaps in HIV service delivery, but have seen little application in the setting of stigma reduction, in part due to a paucity of instruments to reliably measure stigma and evaluate the impact of QI interventions. However, with the development of brief, reliable measurement tools by Nyblade et $a l,{ }^{3}$ and their adaptation to Thailand and surrounding countries, ${ }^{182425}$ the application of a QI approach offers a novel, scalable means to implement stigma-reduction interventions in the healthcare setting, motivated by the aim of translating what is known about the core drivers of stigma into evidence-informed, practice-based action and population-level impact. This approach is informed by successes from prior theory-informed work implementing HIV quality programming in low-income and middle-income country contexts, ${ }^{16}$ whereby sustained achievement of desired outcomes is achieved by building the capacity of national-level, district-level and facility-level cadres to 


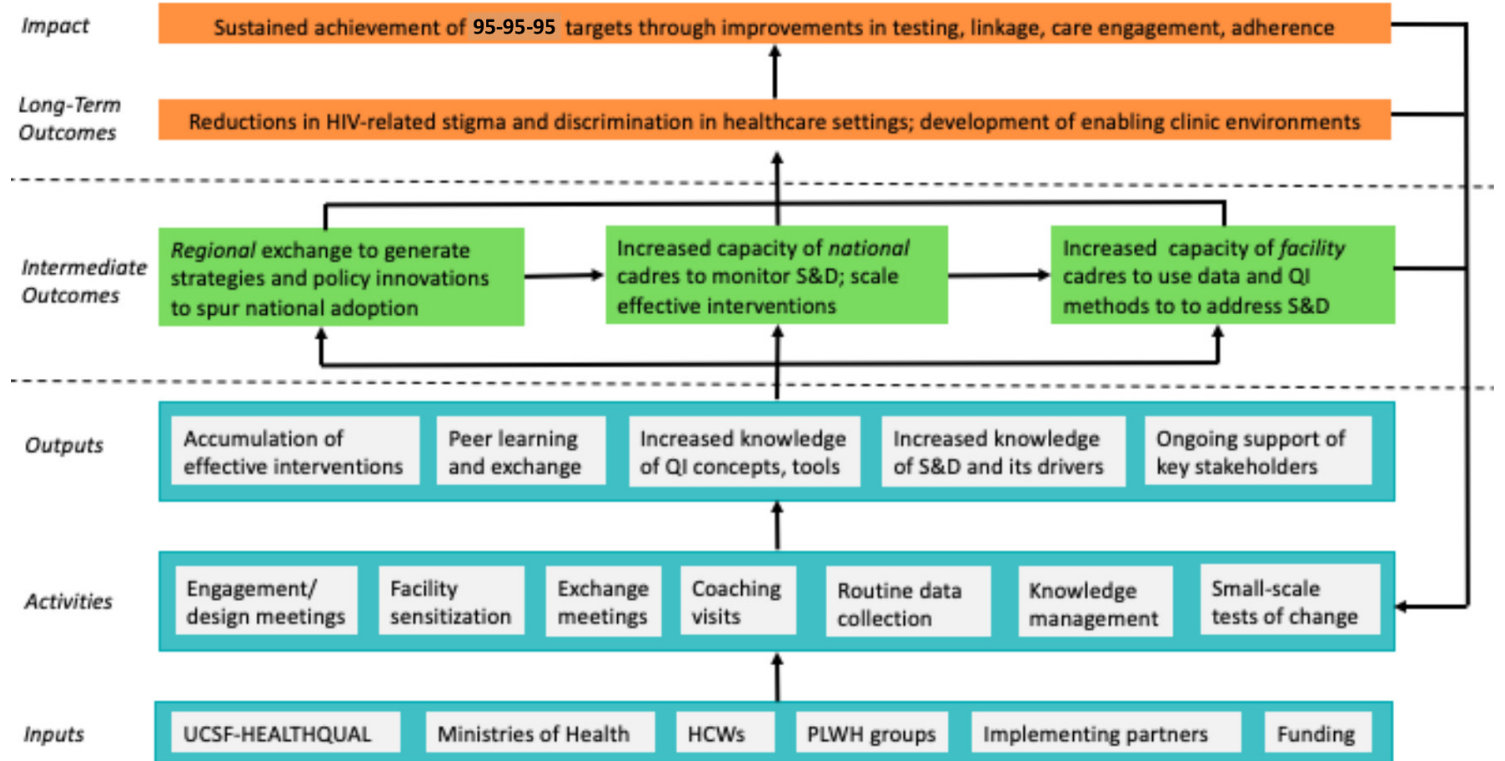

Figure 1 Theory of action - linking QI capacity building to reduction of S\&D. HCWs, healthcare workers; PLWH, people living with HIV; QI, quality improvement; S\&D, stigma and discrimination.

continuously monitor and improve the quality of healthcare service delivery (figure 1).

\section{Network indicators}

At the launch of Network activities, teams from participating countries led by national HIV programmes and comprising stakeholders from civil society organisations, implementing HIV clinics, and relevant implementing partners, selected eight common indicators using a modified Delphi procedure ${ }^{26}$ from a validated instrument of HIV-related S\&D in healthcare settings developed by Srithanaviboonchai et al. ${ }^{18}$ The instrument, which was first adapted from an internationally validated tool $^{3}$ to the Thai context, ${ }^{18}$ was also adapted in Lao People's Democratic Republic ${ }^{24}$ and Vietnam ${ }^{25}$ with only minor changes. Field testing and validation of the instrument was also completed in Cambodia. The eight common indicators are collected by random sampling of clinical and non-clinical HCWs both within and outside HIV clinics on a regular basis using a web-based mobile application, and span the four immediately actionable drivers of HIV-related S\&D (fear of contracting HIV in the workplace, attitudes towards PLWH, awareness of S\&D, institutional environment) (table 1). In addition to surveys of HCWs, participating facilities also collect a common set of indicators on patient experience, which were developed by Network participants through a formal prioritisation exercise and adopted through consensus. ${ }^{26}$ These data are supplemented by analyses of patient feedback from other sources, such as comment boxes, patient fora, exit interviews and community advisory boards. Alongside insights from HCWs, data from PLWH are routinely gathered and analysed to develop S\&D-reduction interventions that are acceptable to PLWH, and informed by their perceptions and experiences of S\&D in the healthcare setting. In addition, findings from clinical performance data (eg, care engagement, viral suppression) may be used to supplement findings from HCWs and PLWH data to drive prioritisation of interventions.

\section{Peer learning and exchange}

By design, the Network seeks to foster peer learning and exchange at multiple levels of implementation (figure 2). At the regional level, multi-country exchange meetings are convened on a quarterly basis with country teams to discuss successes and challenges to implementation of S\&D QI activities in national programmes. As part of these meetings, attendees hear presentations from content experts on QI methods and implementation, HIV-related S\&D measurement, and approaches to $\mathrm{S} \& \mathrm{D}$ reduction. Teams from participating countries also present quarterly updates on implementation progress of S\&D QI activities, with an emphasis on national-level results of S\&D measurement, successfully implemented QI interventions to reduce S\&D at the facility level, approaches to involvement of PLWH organisations in QI activities and plans for scale up. At the national level, participating sites are convened by Ministries of Health on a routine basis to exchange implementation challenges and tested interventions, and spur adoption of S\&D-reduction approaches with proven effectiveness. Documentation of implemented interventions is facilitated by HEALTHQUAL, and shared with Network participants through meetings and production of 'Spotlight' pieces on facility-level implementation.

\section{Selection and support of participating facilities}

Participating sites are selected by Ministries of Health on the basis of country-specific HIV epidemic control priorities. With mentorship and support from implementing partners, national and provincial ministries of health, and technical partners, teams from selected facilities receive training in QI concepts and methods (eg, root cause 
Table 1 Network indicators to assess HIV-related S\&D among HCWs and patient experience among PLWH

\begin{tabular}{ll}
\hline HCWs & Indicator \\
\hline Domain & Do you typically wear double gloves when providing care or services to a patient living with \\
Fear of contracting HIV in & Do you typically avoid physical contact when providing care or services to a patient living with \\
the workplace & HIV? \\
& How worried would you be about getting HIV if you drew blood from a patient living with HIV? \\
Attitudes towards PLWH & Do you strongly agree, agree, disagree or strongly disagree that women living with HIV should \\
Awareness of S\&D & In the past 3 months, have you observed HCWs unwilling to care for a patient living with or \\
& thought to be living with HIV in your health facility? \\
& In the past 3 months, have you observed HCWs providing poorer quality of care to a patient \\
& living with or thought to be living with HIV in your health facility? \\
Institutional environment & Do you strongly agree, agree, disagree or strongly disagree that there are adequate supplies in \\
& your facility that reduce your risk of being infected with HIV? \\
& True/False: Your facility has written guidelines to protect patients living with HIV from \\
& discrimination.
\end{tabular}

PLWH

Indicator

- Was information about your health explained clearly?

- Was the clinic welcoming and friendly?

- Were you treated with respect during your visit?

-Were privacy and confidentiality observed?

- Did you experience discrimination from a healthcare provider or other staff member?

- Were you involved with decision-making about your care and treatment?

- Did your provider spend enough time with you during your visit?

HCWs, healthcare workers; PLWH, people living with HIV; S\&D, stigma and discrimination.

analysis, Plan-Do-Study-Act cycles, QI projects, process mapping, Pareto analysis) with special attention devoted to how these concepts and methods are readily applicable to $\mathrm{S} \& \mathrm{D}$ reduction (box 1 ). Teams also receive training on the four immediately actionable drivers of S\&D in the healthcare setting and their common manifestations, and are instructed in the use of web-based data collection platforms. While the specific content and length of these trainings vary by country, they are all embedded into ongoing HIV quality programming activities and, wherever possible, make use of existing funding streams and QI and S\&D-reduction curricula. On-site coaching and mentoring, a well-documented enabler of QI implementation, ${ }^{27} 28$ is provided by Ministries of Health and implementing partners (Cambodia, Vietnam) to guide sites in the analysis of data from HCWs and PLWH, and the development and testing of S\&D-reduction interventions to address identified areas for improvement.

\section{Progress and next steps}

To date, five multi-country exchange meetings have been convened. QI activities are ongoing in 83 facilities across 29 provinces in Cambodia, Lao People's Democratic Republic, Thailand and Vietnam, with baseline data collection completed in all sites, and follow-up data collection currently underway. Across participating facilities, 27 strategies and interventions are currently being tested, and are presented in table 2 according to domains adapted from the Chronic Care $\mathrm{Model}^{29}$ and the WHO framework of people-centred health services. ${ }^{30}$ These frameworks have been widely used to target QI activities across domains that have been shown to promote longterm engagement in care for chronic conditions, such as HIV. Plans to scale-up S\&D QI activities have been developed by all participating countries, and each country has developed mechanisms through which to support peer learning among participating facilities, including in-person meetings, web-based documents (eg, Google documents) and mobile communication applications (eg, LINE, WhatsApp, ZOOM). As of May 2019, implementation of activities is ongoing, with additional multicountry exchange meetings and continuous measurement planned through fiscal year 2019.

\section{LESSONS LEARNED}

As national HIV programmes move to operationalise global aims to end HIV-related S\&D in healthcare settings, application of a $\mathrm{QI}$ approach to $\mathrm{S} \& \mathrm{D}$ reduction represents a novel means to ensure efforts to achieve these aims are scalable, tailored to context, and ingrained in organisational practice and policy. Lessons learnt through 2 years of Network implementation, and iterative discussions among participating teams as part 


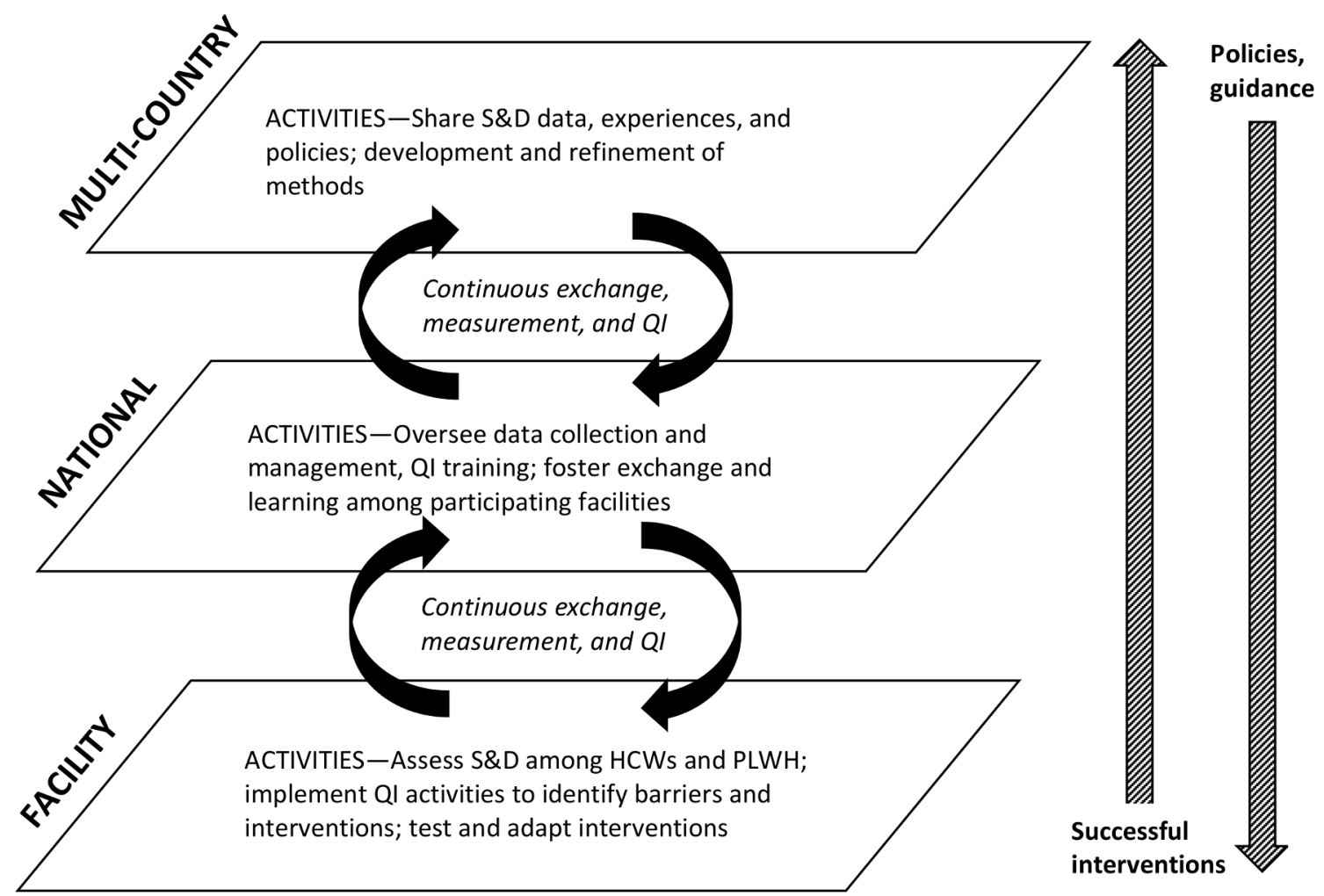

Figure 2 Network design-fostering multiple layers of intervention and exchange. HCWs, healthcare workers; PLWH, people living with HIV; QI, quality improvement; S\&D, stigma and discrimination.

of multi-country exchange meetings, offer programme a suite of best practices for planning, organising and implementing S\&D-reduction activities in the healthcare setting.

\section{Pair knowledge-building interventions with system-level changes}

Addressing deficits in knowledge among HCWs related to HIV, its transmission, and myriad manifestations and impacts of S\&D have been the core of most S\&D-reduction interventions that have been evaluated to date. ${ }^{11}$ While knowledge is a key driver of stigma in the healthcare setting, improvements in knowledge do not always translate into sustained change. To be effective, interventions that attempt to change individual behaviours must also carefully consider the organisational and cultural contexts from which these individuals, and their behaviours, derive meaning and support. ${ }^{31}$ In embracing a QI approach, this initiative has sought to move beyond inputs, such as knowledge of individual HCWs, and facility policies, such as bills of rights and codes of conduct, to consider the systemic processes through which these inputs are translated into processes that lead, in turn, to the outcome of reduced S\&D. System-level changes, like reorganisation of clinic flow, improving privacy, structured interactions between HCWs and PLWH, cultivation of a welcoming, respectful clinic environment, routine pre-service and in-service assessments of S\&D, and evidence-informed responses to these assessments, represent effective ways to bridge the gap between knowledge and action, and support the development of an organisational culture in which S\&D-reduction activities are normalised, expected, and championed as a central component of quality of care.

\section{Engage all HCWs in S\&D-reduction activities, not just those in HIV clinics}

As integrated HIV care delivery models become commonplace in Southeast Asia and elsewhere, there is a growing need to ensure that care delivered to PLWH outside of stand-alone HIV clinics is free of S\&D. Integration of clinic services has been shown to reduce stigma associated with care seeking among PLWH in some settings, ${ }^{32}$ but in others, the result has been the opposite. ${ }^{33}$ Integration of clinic space and patient flow can assist to normalise care-seeking for PLWH, but without changes to organisational practices that 'mark' PLWH-such as dedicated equipment, patient charts and queuesstigma may persist. ${ }^{34}$ In recognition of these challenges, the Network embraced a facility-wide approach to implementation by engaging all HCWs who may have regular contact with PLWH-including nurses, physicians, pharmacists, laboratory technicians, registration staff, porters, cleaners, security staff and hospital leadership. Through this approach, HCWs beyond the HIV clinic are sensitised to S\&D, enabling the consideration of ways through which processes in other hospital departments may fuel S\&D. Limited human and material resources represent a commonly cited challenge to facility-wide approaches to $\mathrm{S} \& \mathrm{D}$ reduction, particularly among those that involve 


\section{Box 1 Application of QI methods to S\&D reduction}

\section{Root Cause Analysis (RCA)}

RCA involves the exploration of the causes of a problem or gap in service delivery. In the context of Network activities, RCA enables participating teams to understand how drivers in their local setting, such as facility-specific policies, processes and resources, contribute to the emergence and perpetuation of a complex phenomenon like stigma. Through RCA, and use of methods such as Ishikawa diagrams and driver diagrams, teams develop a 'theory of action' that identifies levers-root causes-to target for intervention.

\section{Process mapping}

Routine processes-such as patient flow during a clinic visit-are complex, and often assumed to be unchangeable. Contrary to this assumption, these processes are usually the products of deliberate design, and can be modified by mapping out key steps in the process and identifying areas for improvement. As part of S\&D Ql activities, process mapping has been used to characterise specific areas in the hospital where experiences of S\&D experienced by PLWH are particularly common, and where focused intervention may be indicated.

\section{PDSA (Shewhart) cycles}

The PDSA cycle is a structured approach for identifying, testing and adopting improvement interventions. When applied to S\&D, PDSA cycles assist participating teams in prioritising changes that are likely to produce corresponding reductions in S\&D. In the 'Plan' phase, teams develop the intervention to be tested. As part of the 'Do' phase, the selected intervention is implemented on a limited scale. In the 'Study' phase, the results of implementation are analysed to determine the effectiveness of the intervention in reducing S\&D. In the final 'Act' phase, teams decide whether to abandon, adapt or adopt the intervention. Through this iterative testing of changes, teams implement interventions that are uniquely adapted to their local setting based on evidence gathered during PDSA cycles.

PDSA, Plan-Do-Study Act; PLWH, people living with HIV; QI, quality improvement; RCA, root cause analysis; S\&D, stigma and discrimination.

participatory trainings. ${ }^{35}$ Disaggregating data on HCW S\&D and patient experience by service delivery area and cadre, and prioritising interventions using QI methods, maximises the impact of a facility-wide approach amid resource constraints. In addition, leveraging existing platforms to exchange information and plans related to S\&D reduction, such as grand rounds, weekly staff meetings, and PLWH support groups, can further drive routinisation of S\&D-reduction activities and enable the active engagement of all HCWs.

\section{Involve PLWH and communities as active partners in efforts to reduce S\&D}

In planning and implementation of S\&D-reduction activities, the early, continuous and active participation of PLWH is vital, not only as part of the ethical imperative that patients play an active role in decision-making that directly impacts their care, but as a means to empower PLWH to confront S\&D and build demand for people-centred services. In participating countries, people-centred care models are gaining traction, but their implementation remains uneven, in part due to a lack of explicit guidance on how to operationalise these models in routine practice. To speed their adoption, the Network has promoted routine solicitation of patient feedback and involvement-through surveys, comment boxes, exit interviews, community advisory boards and other mechanisms-as a vital component of S\&D-reduction efforts. To best understand the nature of HIV-related S\&D, survey and experience data need to be further analysed and unpacked through disaggregation of data according to key population and behavioural characteristics. To address underlying drivers of HIV-related stigma, a clearer understanding of provider attitudes and beliefs is needed that requires the elicitation of patient characteristics and behaviours that can be used to better understand where the roots of stigma lie so that they can be addressed most effectively. Once the systems are in place to capture data on HIV-related S\&D, these can be augmented to include additional information related to sexual orientation and gender identity, sex work, injection drug use and migrant status. Collection of feedback is a key step to engage PLWH and communities that should be followed by their involvement in co-creating interventions. 'Contact strategies', in which PLWH participate in the delivery of S\&D-reduction interventions, can be effective in promoting empathy, empowering PLWH to demand action, buffering the effects of internalised stigma, and deconstructing HIV-related stereotypes, ${ }^{12}$ and should therefore be promoted in the development of interventions.

\section{Link S\&D-reduction activities to cross-sectoral efforts to improve quality of care}

Ideally, health systems should be designed to ensure that health outcomes are optimised and distributed equitably and efficiently, and that users of health systems receive care that is competent, responsive to their needs, preferences, and identities, and deserving of their ongoing confidence and trust. ${ }^{36}$ As an impediment to achievement of these aspirations, HIV-related S\&D in the healthcare setting not only represents a potent barrier to reaching 95-95-95 targets but also frustrates broader efforts to expand high-quality universal health coverage, particularly in low-income and middle-income country contexts. In its direct engagement of Ministries of Health, this initiative offers a lever by which to stimulate development of policy changes and integration of S\&D reduction into national quality programming that seek to disassemble barriers, such as S\&D, that hinder effective access to universal health coverage, including HIV prevention and treatment services. Recognising that S\&D-reduction activities are applicable beyond disease-specific programming-and focusing engagement at both the national-level and facility-level-assists in building the expectation that $\mathrm{S} \& \mathrm{D}$ reduction is not a time-bound project, but is central to the mandate for health systems to assure and improve the quality of service delivery. Transparently relating S\&D-reduction activities to this obligation offers 


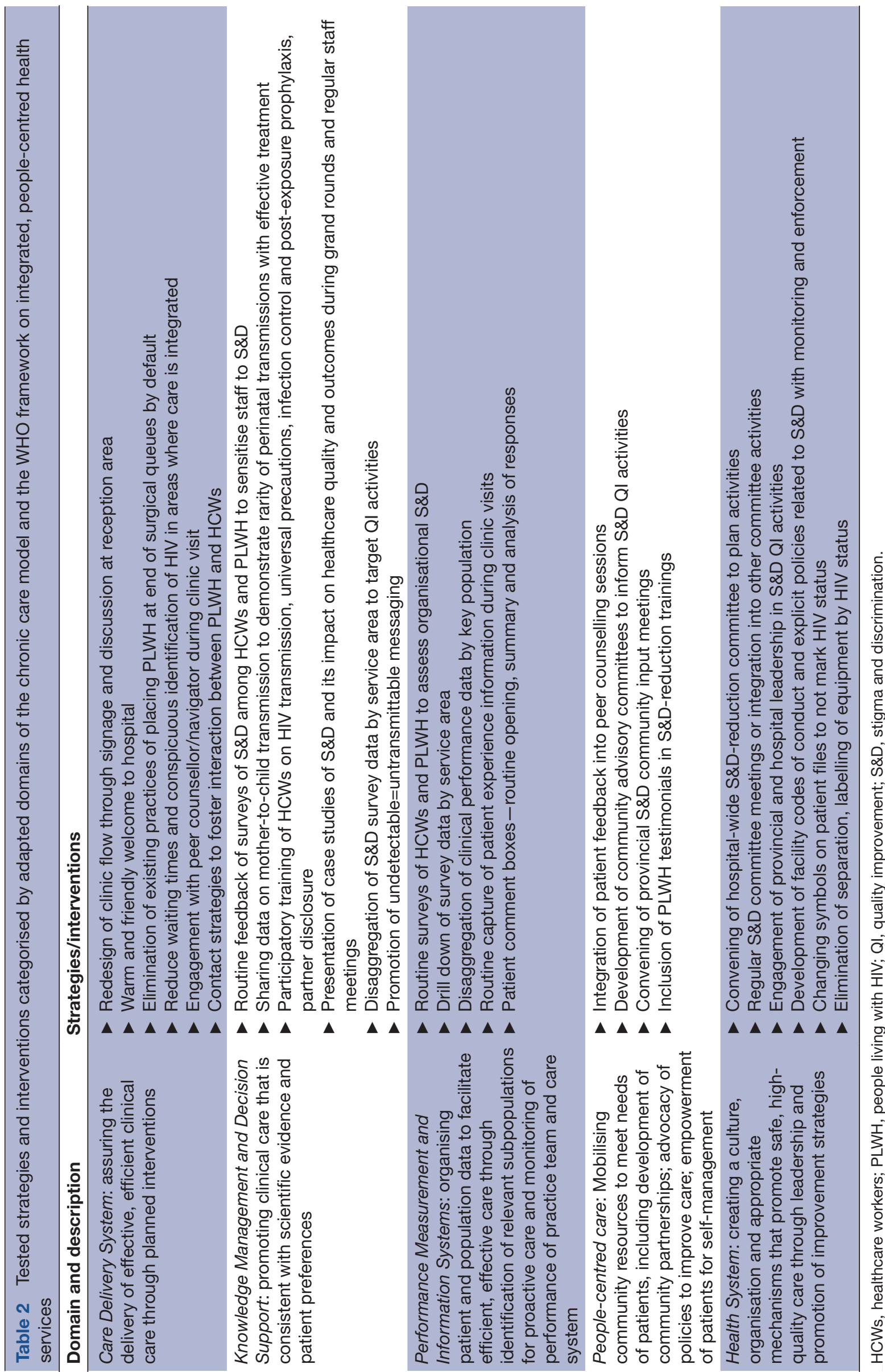

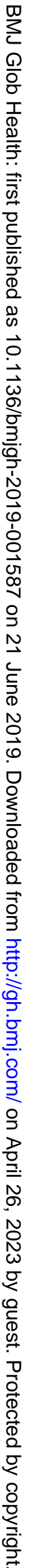


a shared vision and approach that can be leveraged to generate stakeholder buy-in, secure human and material resources, prevent duplication of efforts, and support the sustainability of activities.

\section{CONCLUSION}

As a powerful deterrent to care seeking and engagement among PLWH and key populations at high risk of HIV acquisition, HIV-related S\&D in the healthcare setting impedes global efforts to end the epidemic within the next decade. Despite the availability of tools and interventions to measure and address $\mathrm{S} \& \mathrm{D}$, their integration into routine HIV programming and quality of care activities remains a challenge. Ministry-led application of QI methods to S\&D reduction offers an avenue by which to link S\&D-reduction activities to ongoing efforts to improve quality of care, and to ensure that these activities are implemented as organisational interventions in healthcare facilities, guided by data from HCWs and patient experience, tailored to local contexts, sensitive of available resources and encompassing of individual-level and organisation-level drivers of stigma. This work demonstrates the feasibility of such an approach, and offers insights on how to implement these activities as part of routine components of service delivery in healthcare organisations to advance progress towards achievement of improved individual health outcomes, epidemic control targets and health equity.

Present affiliations The present affiliation of Daniel J Ikeda is: Harvard School of Medicine, MA, Boston, United States.

Acknowledgements The authors are grateful to the participants of the Southeast Asia Stigma Reduction QI Learning Network for their continuing dedication and engagement in this work. The authors also acknowledge the support of Harold Phillips, Tracey Gantt, Katie 0'Connor, and Suzanne Jed.

Contributors DJI and BDA conceived the initial idea for the manuscript. DJI and BDA wrote the first draft. All authors provided critical feedback and approved the final submission.

Funding This work was supported by the US President's Emergency Plan for AIDS Relief as part of the Health Resources and Services Administration's Quality Improvement Capacity for Impact Project award \#U1NHA08599. Its contents do not necessarily reflect the views of the US Government. Publication made possible in part by support from the UCSF Open Access Publishing Fund.

Competing interests None declared.

Patient consent for publication Not required.

Provenance and peer review Not commissioned; externally peer reviewed.

Data availability statement № additional data are available.

Open access This is an open access article distributed in accordance with the Creative Commons Attribution Non Commercial (CC BY-NC 4.0) license, which permits others to distribute, remix, adapt, build upon this work non-commercially, and license their derivative works on different terms, provided the original work is properly cited, appropriate credit is given, any changes made indicated, and the use is non-commercial. See: http://creativecommons.org/licenses/by-nc/4.0/.

\section{REFERENCES}

1. Joint United Nations Program on HIV/AIDS. UNAIDS data 2018, 2018. Available: https://www.unaids.org/sites/default/files/media asset/unaids-data-2018_en.pdf

2. Joint United Nations Program on HIV/AIDS. Confronting discrimination: overcoming HIV-related stigma and discrimination in healthcare settings and beyond, 2017. Available: https:// www.unaids.org/sites/default/files/media_asset/confrontingdiscrimination_en.pdf

3. Nyblade L, Jain A, Benkirane M, et al. A brief, standardized tool for measuring HIV-related stigma among health facility staff: results of field testing in China, Dominica, Egypt, Kenya, Puerto Rico and St. Christopher \& Nevis. J Int AIDS Soc 2013;16.

4. Gesesew HA, Tesfay Gebremedhin A, Demissie TD, et al. Significant association between perceived HIV related stigma and late presentation for HIV/AIDS care in low and middle-income countries: a systematic review and meta-analysis. PLoS One 2017; 12:e0173928.

5. Katz IT, Ryu AE, Onuegbu AG, et al. Impact of HIV-related stigma on treatment adherence: systematic review and meta-synthesis. J Int AIDS Soc 2013;16.

6. McMahon SA, Kennedy CE, Winch PJ, et al. Stigma, facility constraints, and personal Disbelief: why women Disengage from HIV care during and after pregnancy in Morogoro region, Tanzania. AIDS Behav 2017;21:317-29.

7. Joint United Nations Program on HIV/AIDS. Agenda for zero discrimination in health-care settings, 2017. Available: https://www. unaids.org/sites/default/files/media_asset/2017ZeroDiscriminati onHealthCare.pdf

8. Association of Southeast Asian Nations. ASEAN Declaration of commitment on HIV and AIDS: Fast-Tracking and sustaining HIV and AIDS responses to end the AIDS epidemic by 2030, 2016. Available: https://asean.org/wp-content/uploads/2016/09/Final-Endorsed_ ASEAN-Declaration-on-Ending-AIDS_2016.pdf

9. Alexandra Marshall S, Brewington KM, Kathryn Allison M, et al. Measuring HIV-related stigma among healthcare providers: a systematic review. AIDS Care 2017;29:1337-45.

10. Feyissa GT, Lockwood C, Woldie M, et al. Reducing HIV-related stigma and discrimination in healthcare settings: a systematic review of quantitative evidence. PLoS One 2019;14:e0211298.

11. Mak WWS, Mo PKH, Ma GYK, et al. Meta-analysis and systematic review of studies on the effectiveness of HIV stigma reduction programs. Soc Sci Med 2017;188:30-40.

12. Nyblade L, Stockton MA, Giger K, et al. Stigma in health facilities: why it matters and how we can change it. BMC Med 2019;17.

13. Stangl AL, Lloyd JK, Brady LM, et al. A systematic review of interventions to reduce HIV-related stigma and discrimination from 2002 to 2013: how far have we come? J Int AIDS Soc 2013;16.

14. Hewko SJ, Cummings GG, Pietrosanu M, et al. The impact of quality assurance initiatives and workplace policies and procedures on HIV/AIDS-Related stigma experienced by patients and nurses in regions with high prevalence of HIV/AIDS. AIDS Behav 2018;22:3836-46.

15. Pescosolido BA, Martin JK. The stigma complex. Annu Rev Sociol 2015;41:87-116.

16. Bardfield J, Agins B, Akiyama M, et al. A quality improvement approach to capacity building in low- and middle-income countries. AIDS 2015;29(Suppl 2):S179-S186.

17. Proctor EK, Powell BJ, McMillen JC. Implementation strategies: recommendations for specifying and reporting. Implementation Sci 2013;8.

18. Srithanaviboonchai K, Stockton M, Pudpong N, et al. Building the evidence base for stigma and discrimination-reduction programming in Thailand: development of tools to measure healthcare stigma and discrimination. BMC Public Health 2017;17.

19. Vorasane S, Jimba M, Kikuchi K, et al. An investigation of stigmatizing attitudes towards people living with HIV/AIDS by doctors and nurses in Vientiane, Lao PDR. BMC Health Serv Res 2017; 17 .

20. Philbin MM, Hirsch JS, Wilson PA, et al. Structural barriers to HIV prevention among men who have sex with men (MSM) in Vietnam: diversity, stigma, and healthcare access. Plos One 2018;13:e0195000.

21. Yi S, Chhoun P, Suong S, et al. AIDS-related stigma and mental disorders among people living with HIV: a cross-sectional study in Cambodia. PLoS One 2015;10:e0121461.

22. Pulerwitz J, Oanh KTH, Akinwolemiwa D, et al. Improving hospitalbased quality of care by reducing HIV-related stigma: evaluation results from Vietnam. AIDS Behav 2015;19:246-56.

23. Batalden PB, Davidoff $F$. What is "quality improvement" and how can it transform healthcare? Qual Saf Health Care 2007;16:2-3.

24. Philavong B, Chariyalertsak S, Srithanaviboonchai K, et al. A Study on Measuring HIV-Related Stigma and Discrimination in Health Care Settings in Lao People's Democractic Republic. RTI International, 2017.

25. Dzung N. Measurement of HIV-related stigma and discrimination in health care facilities in Ho Chi Minh City. Presented at the: 
Asia regional consultation on addressing HIV-related stigma and discrimination in healthcare settings. Bangkok, Thailand, 2017.

26. Campbell SM, Braspenning J, Hutchinson A, et al. Research methods used in developing and applying quality indicators in primary care. BMJ 2003;326:816-9.

27. Cosimi LA, Dam HV, Nguyen TQ, et al. Integrated clinical and quality improvement coaching in son La Province, Vietnam: a model of building public sector capacity for sustainable HIV care delivery. BMC Health Serv Res 2015;15.

28. Manzi A, Hirschhorn LR, Sherr K, et al. Mentorship and coaching to support strengthening healthcare systems: lessons learned across the five population health implementation and training partnership projects in sub-Saharan Africa. BMC Health Serv Res 2017;17(S3).

29. Wagner EH, Austin BT, Von Korff M. Organizing care for patients with chronic illness. Milbank Q 1996;74:511-44.

30. World Health Organization. Framework on integrated, PeopleCentred health services, 2016. Available: http://apps.who.int/gb/ ebwha/pdf_files/WHA69/A69_39-en.pdf?ua=1\&ua=1

31. Bosk CL, Dixon-Woods M, Goeschel CA, et al. Reality check for checklists. Lancet 2009;374:444-5.
32. Topp SM, Chipukuma JM, Chiko MM, et al. Integrating HIV treatment with primary care outpatient services: opportunities and challenges from a scaled-up model in Zambia. Health Policy Plan 2013;28:347-57.

33. Stockton MA, Giger K, Nyblade L. A scoping review of the role of HIV-related stigma and discrimination in noncommunicable disease care. Plos One 2018;13:e0199602.

34. Bond V, Nomsenge S, Mwamba M, et al. "Being seen" at the clinic: Zambian and South African health worker reflections on the relationship between health facility spatial organisation and items and HIV stigma in 21 health facilities, the HPTN 071 (PopART) study. Health \& Place 2019;55:87-99.

35. Nyblade L, Srinivasan K, Mazur A, et al. HIV stigma reduction for health facility staff: development of a Blended- learning intervention. Front Public Health 2018;6.

36. Kruk ME, Gage AD, Arsenault C, et al. High-quality health systems in the sustainable development goals era: time for a revolution. Lancet Glob Health 2018;6:e1196-252. 